\title{
Editorial: Molecular Components of Store-Operated Calcium Entry in Health and Disease
}

\author{
Joanna Gruszczynska-Biegala ${ }^{1 * t}$, Francisco Javier Martin-Romero ${ }^{2 \dagger}$, Tarik Smani ${ }^{3,4 \dagger}$ and \\ Agnese Secondo ${ }^{5 t}$ \\ 1 Laboratory of Molecular Biology, Mossakowski Medical Research Institute, Polish Academy of Sciences, Warsaw, Poland, \\ ${ }^{2}$ Institute of Molecular Pathology Biomarkers, University of Extremadura, Badajoz, Spain, ${ }^{3}$ Group of Cardiovascular \\ Pathophysiology, Institute of Biomedicine of Seville, University Hospital of Virgen del Rocío, University of Seville, CSIC, Seville, \\ Spain, ${ }^{4}$ Department of Medical Physiology and Biophysics, University of Seville, Seville, Spain, ${ }^{5}$ Department of \\ Neuroscience, "Federico II" University of Naples, Naples, Italy
}

Keywords: SOCE, STIM1, STIM2, TRP channels, ORAI

\section{Editorial on the Research Topic}

\section{Molecular Components of Store-Operated Calcium Entry in Health and Disease}

Store-Operated Calcium Entry (SOCE) is a ubiquitous $\mathrm{Ca}^{2+}$ influx mechanism first described in 1986 (Putney, 1986). This conserved mechanism results from the interaction between the tetraspanning ORAI1 channel located in plasma membrane and the unique endoplasmic reticulum (ER) $\mathrm{Ca}^{2+}$ sensor, stromal interaction molecule 1 (STIM1), via their respective intracellular domains (Roos et al., 2005; Csutora et al., 2008). Moreover, there are two homologs of ORAI1, namely ORAI2 and ORAI3, all generating SOCE with the same mechanism.

Molecularly, upon ER $\mathrm{Ca}^{2+}$ depletion, STIM1 senses the filling state of ER with its Nterminus characterized by a low affinity for $\mathrm{Ca}^{2+}$ ion (200 nM-600 microM). After dimerization, STIM1 diffuses to the plasma membrane regions where it interacts with ORAI1 inducing SOCE through Calcium Release-Activated Calcium (CRAC) channel opening. This event may mediate a localized increase in the intracellular $\mathrm{Ca}^{2+}$ concentration useful to recharge $\mathrm{ER}$ of $\mathrm{Ca}^{2+}$ content by sarco/endoplasmic reticulum $\mathrm{Ca}^{2+}$-ATPase (SERCA) intervention. Besides STIM1, also STIM2 may induce activation of ORAI1 (Brandman et al., 2007) but with a weak $\mathrm{Ca}^{2+}$ entry that is nevertheless able to trigger NFAT1 activation (Son et al., 2020).

Accumulated evidence suggests that SOCE dysfunction may produce $\mathrm{Ca}^{2+}$ dyshomeostasis in both excitable and non-excitable cells, thus participating to the pathogenesis of a large spectrum of diseases most of which are due to the modification of ORAI1/STIM1 interaction in consequence of changes in their expression or following a disruption of SOCE machinery. However, genetic modification of the two major players could also occur. For instance, loss of function mutations of ORAI1 and STIM1/STIM2 abolishes SOCE, thus causing autoimmunity and severe combined immunodeficiency (SCID)-like diseases. In contrast, autosomal dominant gain-of-function mutations in ORAI1 and STIM1 determine a sustained increase in CRAC and SOCE causing a large spectrum of diseases like Stormorken syndrome and nonsyndromic tubular aggregate myopathy (TAM) (Lacruz and Feske, 2015). In this respect the study by Conte et al. shows some of the adaptive or compensatory mechanisms able to counteract the genetic-encoded $\mathrm{Ca}^{2+}$ dyshomeostasis and describes the alteration in the differentiation process of muscle cells deriving from TAM-patients carrying STIM1 L96V mutation.

Mechanistically, mutations in the transmembrane domains of the pore region of ORAI channels may produce SOCE alterations. Of note, the elucidation of the isoform-specific mutations may 
provide useful targets for selective drug development. In this context, the study by Tiffner et al. shows the molecular consequences of the enhanced hydrophobicity along TM3 of ORAI1 or ORAI3 structure on the gain-of-function of SOCE and CRAC currents.

In this Research Topic, two manuscripts review the role of ORAI1 in cardiovascular remodeling and heart failure. The first article by Luo et al. provides an overview of the new role of ORAI1-mediated SOCE in the maladaptive cardiac hypertrophy and heart failure. The review discusses the current knowledge regarding the involvement of ORAI1 in hypertrophic development induced by neurohormonal stimulation in vitro or in vivo, using transgenic mice subjected to different procedures that induce cardiac hypertrophy and consequently heart failure.

The other review article by Shawer et al. provides a broad perspective of the role ORAI1-mediated vascular smooth muscle cell (VSMC) switching from contractile to synthetic phenotypes, a critical step for their proliferation and migration. Authors focus on the involvement of ORAI1 in the pathological vascular remodeling related to atherosclerosis, neointimal hyperplasia and restenosis. They also discuss the potential mode of action of a large list of SOCE inhibitors examined in different cell lines.

Both reviews consider that new pharmacological selective inhibitors are valuable tools to study the role of SOCE in health and disease, which might pave the way for the development of therapeutic ORAI1 inhibitors to mitigate pathologic cardiac and vascular remodeling.

Galeano-Otero et al. in an original article related to the circulatory system highlight for the first time the role of SARAF, SOCE-associated regulatory factor, in the activation of endothelial cells and angiogenesis. The study nicely demonstrates that SOCE participates in several steps of angiogenesis, such as endothelial cell proliferation and migration, tube formation, and sprouting, and shows that SARAF co-localizes and interacts with ORAI1, which might sustain an enhanced $\mathrm{Ca}^{2+}$ entry in these highly proliferative cells during angiogenesis.

Since the discovery of the molecular components of SOCE, including the STIM, ORAI and TRP proteins, a growing number of publications describe their functions in the healthy and diseased central nervous system (CNS) (Serwach and Gruszczynska-Biegala, 2019). In this regard, Zhang and $\mathrm{Hu}$ summarize the current literature reporting on the variable expression of STIM, ORAI and TRPC in neurons and glial cells from different parts of the CNS. The authors also provide a systematic overview describing the current understanding of the physiological and pathological role of SOCE and its molecular components in each of these brain regions. The impact of existing therapies on SOCE is also reviewed.

Two additional review articles describe the relationship between Huntington's disease (HD) and SOCE (Latoszek and Czeredys). As an inherited neurodegenerative disorder, $\mathrm{HD}$ is characterized by the loss of $\gamma$-aminobutyric acid (GABA)-ergic medium spiny neurons (MSNs) in the striatum (Vonsattel and DiFiglia, 1998), and SOCE has been shown to be elevated in several $\mathrm{HD}$ models. Czeredys's review provides a comprehensive update on the implications of SOCE,
STIM2, ORAIs and TRPCs in HD pathology using various HD models, including YAC128 mice (HD transgenic model), HD cellular models, and induced pluripotent stem cell-based GABAergic MSNs that are obtained from fibroblasts of adult HD patients. The author further discusses potential drug candidates that may restore normal SOCE and consequently prevent dendritic spine loss in HD. Latoszek and Czeredys review recent data indicating that $\mathrm{HD}$ is also a neurodevelopmental disease, as neuronal cells differentiated from juvenile HD patient-derived iPSCs show deficits in development and adult neurogenesis. Finally, the authors also review different protocols to obtain MSNs and brain organoids as powerful tools to study $\mathrm{HD}$.

Also related to $\mathrm{HD}$, Vigont et al. in an original article, demonstrate the role of STIM2 in the elevated $\mathrm{Ca}^{2+}$ entry in HD cellular models. More specifically, these authors generated MSNs modeling a juvenile form of $\mathrm{HD}$ and show high levels of SOCE using patch-clamp. Upregulation of STIM2 protein expression was also observed, and the shRNA-mediated suppression of STIM2 expression attenuated SOCE. For this reason, Vigont et al. used the anti-HD drug EVP4593 to demonstrate that this drug decreased huntingtin expression and also the expression of STIM2, postulating that STIM2 could be a novel therapeutic target for HD.

In addition to the main components of SOCE, being the STIM and ORAI proteins, an increasing number of proteins have been also reported to play an important role in the STIM-ORAI dependent regulation of SOCE. Serwach and Gruszczynska-Biegala thoroughly review and summarize the current knowledge about STIM protein target molecules, including positive (mGluR, septins, synaptopodin, POST, EB and Golli proteins) and negative (Homer, SARAF, presenilin1, and NEUROD2) regulators and effectors [L-type voltage-operated $\mathrm{Ca}^{2+}$ channels (VOCCs) and receptors such as AMPAR and NMDAR] in the CNS. This review also highlights the importance of the interaction of STIM proteins with their target proteins in pathology, such as hypoxic/ischemic neuronal injury, epilepsy, Alzheimer's, Huntington's and Parkinson's diseases, and in physiological conditions of the CNS.

Finally, in this issue Coronas et al. review the role of $\mathrm{Ca}^{2+}$ channels in neural stem cells (NSCs) and glioblastoma stem cells (GSCs), derived from oncogenic mutations in adult NSCs and responsible for the emergence of malignant brain tumors. The review focuses on the $\mathrm{Ca}^{2+}$ toolkit and its physiological role in GSCs, in NSCs and their progenies to show that stemness is controlled by VOCCs, store-operated $\mathrm{Ca}^{2+}$ channels (SOCs), $\mathrm{IP}_{3} \mathrm{Rs}$ in NSCs, and by VOCCs, nicotinic receptors and TRPVs in GSCs.

In summary, this special issue of Frontiers in Cellular Neuroscience provides a comprehensive overview of the most recent data on the role of SOCE and related proteins: STIMs, ORAIs and others that regulate them, in the physiology and pathology of a variety of cells. It highlights the importance of SOCE and its regulatory proteins as potential drug targets for future therapies against neurodegenerative, cardiovascular or muscular diseases and brain tumors. 


\section{AUTHOR CONTRIBUTIONS}

All authors listed have made a substantial, direct and intellectual contribution to the work, and approved it for publication.

\section{FUNDING}

JG-B was supported by the National Science Centre grant (research project no. 2017/26/E/NZ3/01144), FJM-R was supported by the Spanish Ministry of Science and Innovation (research project BFU2017-82716-P), TS was supported by the Spanish Ministry of Science and Innovation (research project

\section{REFERENCES}

Brandman, O., Liou, J., Park, W. S., and Meyer, T. (2007). STIM2 is a feedback regulator that stabilizes basal cytosolic and endoplasmic reticulum $\mathrm{Ca}^{2+}$ levels. Cell 131, 1327-1339. doi: 10.1016/j.cell.2007.11.039

Csutora, P., Peter, K., Kilic, H., Park, K. M., Zarayskiy, V., Gwozdz, T., et al. (2008). Novel role for STIM1 as a trigger for calcium influx factor production. J. Biol. Chem. 283, 14524-14531. doi: 10.1074/jbc.M709575200

Lacruz, R. S., and Feske, S. (2015). Diseases caused by mutations in ORAI1 and STIM1. Ann. N. Y. Acad. Sci. 1356, 45-79. doi: 10.1111/nyas.12938

Putney, J. W. Jr. (1986). A model for receptor-regulated calcium entry. Cell Calcium 7, 1-12. doi: 10.1016/0143-41608690026-6

Roos, J., DiGregorio, P. J., Yeromin, A. V., Ohlsen, K., Lioudyno, M., Zhang, S., et al. (2005). STIM1, an essential and conserved component of store-operated $\mathrm{Ca}^{2+}$ channel function. J. Cell Biol. 169, 435-445. doi: 10.1083/jcb.200502019

Serwach, K., and Gruszczynska-Biegala, J. (2019). STIM proteins and glutamate receptors in neurons: role in neuronal physiology and neurodegenerative diseases. Int. J. Mol. Sci. 20:2289. doi: 10.3390/ijms20092289

Son, G. Y., Subedi, K. P., Ong, H. L., Noyer, L., Saadi, H., Zheng, C., et al. (2020). STIM2 targets Orail/STIM1 to the AKAP79 signaling complex and confers coupling of $\mathrm{Ca}^{2+}$ entry with NFAT1 activation. Proc. Natl. Acad. Sci.U.S.A. 117, 16638-16648. doi: 10.1073/pnas.1915386117 no. PID2019-104084GB-C22/ AEI/10.13039/501100011033), AS was supported by PRIN2015-Prot. 2015KRYSJN (Italian Ministry of Education and Research), Progetto Speciale di Ateneo CA.04_CDA_n_103 27.03.2019 and Progetto di Ateneo Linea A CdA_54_2020_FRA.

\section{ACKNOWLEDGMENTS}

Editors thank deeply all the authors for their scientific contributions and the reviewers for their support and constructive criticism in refining the manuscripts in this Research Topic.

Vonsattel, J. P., and DiFiglia, M. (1998). Huntington disease. J. Neuropathol. Exp. Neurol. 57, 369-384. doi: 10.1097/00005072-199805000-0 0001

Conflict of Interest: The authors declare that the research was conducted in the absence of any commercial or financial relationships that could be construed as a potential conflict of interest.

Publisher's Note: All claims expressed in this article are solely those of the authors and do not necessarily represent those of their affiliated organizations, or those of the publisher, the editors and the reviewers. Any product that may be evaluated in this article, or claim that may be made by its manufacturer, is not guaranteed or endorsed by the publisher.

Copyright (c) 2021 Gruszczynska-Biegala, Martin-Romero, Smani and Secondo. This is an open-access article distributed under the terms of the Creative Commons Attribution License (CC BY). The use, distribution or reproduction in other forums is permitted, provided the original author(s) and the copyright owner(s) are credited and that the original publication in this journal is cited, in accordance with accepted academic practice. No use, distribution or reproduction is permitted which does not comply with these terms. 